\title{
Symmetry reduction and exact solutions of the non-linear Black-Scholes equation
}

\author{
Oleksii Patsiuk $^{\mathrm{a}, \mathrm{b}, *}$, Sergii Kovalenko ${ }^{\mathrm{c}}$ \\ ${ }^{a}$ Institute of Mathematics, National Academy of Sciences of Ukraine, 3 Tereshchenkivs'ka \\ Str., 01601 Kyiv-4, Ukraine \\ ${ }^{b}$ PrivatBank, 6 Seryozhnikova Str., 14006 Chernihiv, Ukraine \\ ${ }^{c}$ Ardas Group Inc., 77 20-richchya Peremohy Str., 49127 Dnipro, Ukraine
}

\begin{abstract}
In this paper, we investigate the non-linear Black-Scholes equation:

$$
u_{t}+a x^{2} u_{x x}+b x^{3} u_{x x}^{2}+c\left(x u_{x}-u\right)=0, \quad a, b>0, c \geq 0 .
$$

and show that the one can be reduced to the equation

$$
u_{t}+\left(u_{x x}+u_{x}\right)^{2}=0
$$

by an appropriate point transformation of variables. For the resulting equation, we study the group-theoretic properties, namely, we find the maximal algebra of invariance of its in Lie sense, carry out the symmetry reduction and seek for a number of exact group-invariant solutions of the equation. Using the results obtained, we get a number of exact solutions of the Black-Scholes equation under study and apply the ones to resolving several boundary value problems with appropriate from the economic point of view terminal and boundary conditions.
\end{abstract}

Keywords: Black-Scholes equation, symmetry reduction, exact solutions

\section{Introduction}

In modern mathematical finance, the Black-Scholes equation (BSE) is one of the key equations used in option pricing theory. Note that the standard derivative pricing theory is based on the assumption of perfectly liquid markets. In this case, the well studied linear BSE 1, 2 is used. But in recent years much attention is paid to illiquid markets. As noted in [3] (see also [4]), the most comprehensive equation providing the price of a European option is the following non-linear BSE:

$$
u_{t}+\frac{1}{2} \tilde{\sigma}^{2}\left(S, u_{S}, u_{S S}\right) S^{2} u_{S S}+r\left(S u_{S}-u\right)=0, \quad r \geq 0,
$$

\footnotetext{
* Corresponding author.

Email addresses: patsyuck@yahoo.com (Oleksii Patsiuk), kovalenko@imath.kiev.ua
} (Sergii Kovalenko)

Preprint submitted to Communications in Nonlinear Science and Numerical SimulationMarch 15, 2018 
where $u$ is the price of the European option under study, $S$ is the price of the underlying stock, $r$ is the risk-free interest rate, and $\tilde{\sigma}$ is the volatility function.

For modeling illiquid markets, one can use [4]:

1) transaction-cost models with the volatility function of the form ${ }^{1}$

$$
\tilde{\sigma}^{2}=\sigma^{2}\left(1+2 \rho S u_{S S}\right)
$$

2) reduced-form stochastic differential equation (SDE) models with the volatility function

$$
\tilde{\sigma}^{2}=\frac{\sigma^{2}}{\left(1-\rho S u_{S S}\right)^{2}} ;
$$

3) equilibrium (reaction-function) models with the volatility function

$$
\tilde{\sigma}^{2}=\frac{\sigma^{2}\left(1-\rho u_{S}\right)^{2}}{\left(1-\rho u_{S}-\rho S u_{S S}\right)^{2}} .
$$

In all these formulas $\sigma$ is the constant (historical) volatility, and $\rho$ is a parameter modeling the liquidity of the market under study ${ }^{2}$.

Since $\rho$ is often considered to be small, we can replace $\tilde{\sigma}^{2}$ with its first order Taylor approximation around $\rho=0$ in the last two formulas. Thus, for small values of $\rho$ we can restrict our considerations by the transaction-cost models and investigate only the BSE of the form:

$$
u_{t}+\frac{1}{2} \sigma^{2} S^{2} u_{S S}\left(1+2 \rho S u_{S S}\right)+r\left(S u_{S}-u\right)=0, \quad \sigma, \rho>0, r \geq 0 .
$$

The non-linear BSE with $\tilde{\sigma}^{2}$ of the form $\sigma^{2}\left(1+2 \rho S u_{S S}\right)$ is widely used in Financial Mathematics. Note that equation (2) is a partial case of equations (1.1) and (28) considered in [7] and [8, respectively. Equation [2] with $r=0$ was also investigated in [3, 4, 6, 9. In particular, using methods of the Lie group theory, Bobrov [9] find the maximal algebra of invariance of the one, carry out the symmetry reduction and present examples of exact invariant solutions.

Using the notation $a=\frac{1}{2} \sigma^{2}, b=\rho \sigma^{2}, c=r$, and $x=S$, we rewrite 2 in a more convenient form:

$$
u_{t}+a x^{2} u_{x x}+b x^{3} u_{x x}^{2}+c x u_{x}-c u=0, \quad a, b>0, c \geq 0 .
$$

In what follows, we consider only the values of independent variables $t, x$ from the domain $\mathbb{R}_{+} \times \mathbb{R}_{+}$(this is due to the economic sense of these variables). With a view to avoiding cumbersome calculations made by Bobrov in the case $c=0$, we reduce (3) to a simpler form using point transformations of variables.

\footnotetext{
${ }^{1}$ This model was suggested by Çetin, Jarrow, and Protter [5]. Note that in 4 several other transaction-cost models with some different volatility functions are considered.

${ }^{2}$ For $\rho=0$ the market is perfectly liquid (and we have the linear BSE), whereas for $\rho$ large a trade has a substantial impact on the transaction price. For the stock of major U.S. corporations $\rho$ is a small parameter (of the order of $10^{-4}$ ) [6, p. 186].
} 
Having made the group analysis of the obtained equation and built a number of its exact invariant solutions, we transform them into ones of equation (3) using the inverse transformations of variables.

The structure of this article is as follows. In Section 2, using the simplifying point transformations of variables, we reduce the non-linear BSE (3) to a partial differential equation (PDE), which is a special case of an equation from the famous handbook 10. In Section 3, we present the optimal system of the one-dimensional sub-algebras of maximal algebra of invariance (MAI) of the obtained equation, carry out the symmetry reduction, and get a number of exact group-invariant solutions of the one. Returning to BSE (3), we obtain a number of its exact solutions in Section 4. Next, we apply the solution found in Section 4 to solving several BVPs with the governing equation (3). Finally, in Section 6, we briefly sum up the results of this paper.

\section{Simplifying point transformations of variables}

Using the point transformations of variables

$$
\begin{gathered}
\bar{t}=t, \quad \bar{x}=\log \frac{x}{b}, \quad \bar{u}=\frac{b u}{x}+\frac{a}{2} \log \frac{x}{b}-\frac{a^{2}}{4} t \quad(c=0) ; \\
\bar{t}=c t, \quad \bar{x}=\log \frac{c x}{b}-c t, \quad \bar{u}=\frac{b u}{c x}+\frac{a}{2 c} \log \frac{c x}{b}-\frac{a}{2}\left(1+\frac{a}{2 c}\right) t \quad(c>0),
\end{gathered}
$$

we can reduce equation 3 to the equation

$$
u_{t}+\left(u_{x}+u_{x x}\right)^{2}=0
$$

(hereafter we omit the overlines for convenience).

We get an equation of the form $u_{t}=F\left(u_{x}, u_{x x}\right)$. It is known (see [10, Subs. 12.1.1, No. 2]) that the resulting equation admits traveling-wave solution

$$
u(t, x)=u(\xi), \quad \xi=k x+\lambda t
$$

where the function $u(\xi)$ is determined by the autonomous ordinary differential equation (ODE)

$$
F\left(k u_{\xi}, k^{2} u_{\xi \xi}\right)-\lambda u_{\xi}=0,
$$

and a more complicated solution of the form

$$
u(t, x)=c_{1}+c_{2} t+\varphi(\xi), \quad \xi=k x+\lambda t,
$$

where the function $u(\xi)$ is determined by the autonomous ODE

$$
F\left(k \varphi_{\xi}, k^{2} \varphi_{\xi \xi}\right)-\lambda \varphi_{\xi}-c_{2}=0 .
$$

In Section 3 , we find a number of other solutions of equation (6). 


\section{Symmetry reduction and exact solutions of equation (6)}

Using program LIE [11, we obtain that the basis of MAI of equation (6) can be chosen as follows:

$$
X_{1}=-\partial_{x}, \quad X_{2}=-e^{-x} \partial_{u}, \quad X_{3}=\partial_{t}, \quad X_{4}=\partial_{u}, \quad X_{5}=t \partial_{t}-u \partial_{u} .
$$

Non-zero commutators of this operators are:

$$
\left[X_{1}, X_{2}\right]=X_{2}, \quad\left[X_{2}, X_{5}\right]=-X_{2}, \quad\left[X_{3}, X_{5}\right]=X_{3}, \quad\left[X_{4}, X_{5}\right]=-X_{4} .
$$

Hence, our MAI $A$ can be written as a semidirect sum of a one-dimensional algebra and a four-dimensional ideal:

$$
A=\left\{X_{5}\right\} \Subset\left\{X_{1}, X_{2}, X_{3}, X_{4}\right\} .
$$

The ideal is of the type $A_{2.2} \oplus 2 A_{1}$ (here we apply the notations used in [12]). Using this facts and executing the well-known classification algorithm [13, p. 1450], we obtain the following assertion.

Proposition 1. The optimal system of the one-dimensional subalgebras of MAI of equation (6) consists of the following ones: $\left\langle X_{1}\right\rangle,\left\langle X_{2}\right\rangle,\left\langle X_{3}\right\rangle,\left\langle X_{4}\right\rangle,\left\langle X_{5}\right\rangle$, $\left\langle X_{1}+\varepsilon X_{3}\right\rangle,\left\langle X_{1}+\varepsilon X_{4}\right\rangle,\left\langle X_{2}+\varepsilon X_{3}\right\rangle,\left\langle X_{2}+\varepsilon X_{4}\right\rangle,\left\langle X_{3}+\varepsilon X_{4}\right\rangle,\left\langle X_{1}+y\left(\varepsilon_{1} X_{3}+\right.\right.$ $\left.\left.\varepsilon_{2} X_{4}\right)\right\rangle,\left\langle X_{2}+\sin \varphi\left(\varepsilon_{1} X_{3}+\varepsilon_{2} X_{4}\right)\right\rangle,\left\langle X_{5}+z X_{1}\right\rangle,\left\langle X_{5}-X_{1}+\varepsilon X_{2}\right\rangle$, where $\varepsilon= \pm 1$, $\varepsilon_{1}= \pm 1, \varepsilon_{2}= \pm 1, y>0, z \neq 0,-1$, and $0<\varphi<\frac{\pi}{2}$.

First of all, note that the algebras $\left\langle X_{2}\right\rangle,\left\langle X_{4}\right\rangle$, and $\left\langle X_{2}+\varepsilon X_{4} \mid \varepsilon= \pm 1\right\rangle$ do not satisfy the necessary conditions for existence of the non-degenerate invariant solutions. Further, we perform the detailed analysis of invariant solutions, which is based on all other algebras from Proposition 1. The results of our investigation are presented in Tables 1 and 2. Table 1 consists of anzatses generated by the subalgebras and corresponding reduced equations, exact solutions of which (or the first order ODEs, if we could not find their solutions) are given in Table 2 .

Remark 1. Reduced equations 6 and 8 (with $k<0$ ) from Table 1 can have real-valued solutions, only if $\varepsilon=-1$.

\section{Remark 2. In Table 2,}

1) solution 1 is trivial and can be included to solution 2;

2) solution 3 is the traveling-wave one, which can be obtained from (7), if we put $k=1, \lambda=\varepsilon$

3) solution 4 can be obtained from solution 3 , if we put $c_{2}=0$;

4) solution 7 is of the form (8), and one can be obtained, if we put $k=1, \lambda=\frac{1}{k}$, $c_{2}=\varepsilon$ 
Table 1: The symmetry reduction of equation 6)

\begin{tabular}{llll}
\hline No. & Algebra $^{\mathrm{a}}$ & Ansatz & Reduced equation \\
\hline 1 & $\left\langle X_{1}\right\rangle$ & $u=\varphi(t)$ & $\varphi^{\prime}=0$ \\
2 & $\left\langle X_{3}\right\rangle$ & $u=\varphi(x)$ & $\varphi^{\prime \prime}+\varphi^{\prime}=0$ \\
3 & $\left\langle X_{1}+\varepsilon X_{3}\right\rangle$ & $u=\varphi(x+\varepsilon t)$ & $\left(\varphi^{\prime \prime}+\varphi^{\prime}\right)^{2}+\varepsilon \varphi^{\prime}=0$ \\
4 & $\left\langle X_{1}+\varepsilon X_{4}\right\rangle$ & $u=\varphi(t)-\varepsilon x$ & $\varphi^{\prime}=-1$ \\
5 & $\left\langle X_{2}+\varepsilon X_{3}\right\rangle$ & $u=\varphi(x)-\varepsilon t e^{-x}$ & $\left(\varphi^{\prime \prime}+\varphi^{\prime}\right)^{2}-\varepsilon e^{-x}=0$ \\
6 & $\left\langle X_{3}+\varepsilon X_{4}\right\rangle$ & $u=\varphi(x)+\varepsilon t$ & $\left(\varphi^{\prime \prime}+\varphi^{\prime}\right)^{2}+\varepsilon=0$ \\
$7^{\mathrm{b}}$ & $\left\langle X_{1}+k\left(X_{3}+\varepsilon X_{4}\right)\right\rangle$ & $u=\varphi(y)+\varepsilon t$ & $\left(\varphi^{\prime \prime}+\varphi^{\prime}\right)^{2}+\frac{1}{k} \varphi^{\prime}+\varepsilon=0$ \\
$8^{\mathrm{c}}$ & $\left\langle X_{2}+k\left(X_{3}+\varepsilon X_{4}\right)\right\rangle$ & $u=\varphi(x)+\left(\varepsilon-\frac{1}{k} e^{-x}\right) t$ & $\left(\varphi^{\prime \prime}+\varphi^{\prime}\right)^{2}-\frac{1}{k} e^{-x}+\varepsilon=0$ \\
9 & $\left\langle X_{5}\right\rangle$ & $u=t^{-1} \varphi(x)$ & $\left(\varphi^{\prime \prime}+\varphi^{\prime}\right)^{2}-\varphi=0$ \\
$10^{\mathrm{d}}$ & $\left\langle X_{5}+k X_{1}\right\rangle$ & $u=t^{-1} \varphi(y)$ & $\left(\varphi^{\prime \prime}+\varphi^{\prime}\right)^{2}+k \varphi^{\prime}-\varphi=0$ \\
$11^{\mathrm{e}}$ & $\left\langle X_{5}-X_{1}+\varepsilon X_{2}\right\rangle$ & $u=e^{-x}(\varphi(y)-\varepsilon x)$ & $\left(\varphi^{\prime \prime}-\varphi^{\prime}+\varepsilon\right)^{2}-e^{y} \varphi^{\prime}=0$ \\
\hline
\end{tabular}

\footnotetext{
${ }^{\mathrm{a}}$ In this column, $\varepsilon= \pm 1$.

${ }^{\mathrm{b}}$ In this case, $k \neq 0, y=x+\frac{1}{k} t$.

${ }^{\mathrm{c}}$ In this case, $0<|k|<1$.

${ }^{\mathrm{d}}$ In this case, $k \neq 0,-1, y=x+k \log t$.

${ }^{\mathrm{e}}$ In this case, $y=x-\log t$.
}

5) ODE 11 is obtained, if we put in ODE 7 from Table 1

$$
z=-\frac{1}{k} e^{\frac{1}{2} y}, \quad \omega=e^{\frac{1}{2} y} \sqrt{-\left(\varepsilon+\frac{1}{k} \varphi^{\prime}(y)\right)}
$$

and admits the solution in the parametric form (see [14, Subs. 1.3.1, No. 2]):

$$
z=z(\tau), \quad w=\tau \cdot z(\tau)
$$

where $z(\tau)$ is defined as:

a) $z(\tau)=c_{1}\left(\left|2 \tau-1+\sqrt{4 k^{2}+1}\right|^{1-\frac{1}{\sqrt{4 k^{2}+1}}}\left|2 \tau-1-\sqrt{4 k^{2}+1}\right|^{1+\frac{1}{\sqrt{4 k^{2}+1}}}\right)^{-\frac{1}{2}}$,

if $\varepsilon=-1$, and $k \neq 0$;

b) $z(\tau)=c_{1}\left(\left|2 \tau-1+\sqrt{1-4 k^{2}}\right|^{1-\frac{1}{\sqrt{1-4 k^{2}}}}\left|2 \tau-1-\sqrt{1-4 k^{2}}\right|^{1+\frac{1}{\sqrt{1-4 k^{2}}}}\right)^{-\frac{1}{2}}$,

if $\varepsilon=1$, and $0<|k|<\frac{1}{2}$;

c) $z(\tau)=\frac{c_{1}}{2 \tau-1} e^{\frac{1}{2 \tau-1}}$, if $\varepsilon=1$, and $k= \pm \frac{1}{2}$;

d) $z(\tau)=c_{1}\left(\tau^{2}-\tau+k^{2}\right)^{-\frac{1}{2}} e^{-\frac{1}{\sqrt{4 k^{2}-1}} \arctan \frac{2 \tau-1}{\sqrt{4 k^{2}-1}}}$, if $\varepsilon=1$, and $|k|>\frac{1}{2}$;

6) ODE 12 is obtained, if we put in ODE 9 from Table 1

$$
z=\frac{1}{6} \sqrt{\varphi^{3}}, \quad \omega=\frac{1}{2} \varphi^{\prime}
$$

this is the Abel equation of the second kind [14, Subs. 1.3.2]; 
Table 2: The exact group-invariant solutions of equation 6

\begin{tabular}{lll}
\hline No. & Algebra $^{\mathrm{a}}$ & Exact solution or first order ODE \\
\hline 1 & 1 & $u=c_{1}$ \\
2 & 2 & $u=c_{1}+c_{2} e^{-x}$ \\
3 & 3 & $u=c_{1}-\varepsilon(x+\varepsilon t)+4 \delta c_{2} e^{-\frac{1}{2}(x+\varepsilon t)}+\varepsilon c_{2}^{2} e^{-(x+\varepsilon t)}$ \\
4 & 4 & $u=c_{1}-t-\varepsilon x$ \\
5 & 5 & $u=c_{1}+4 \delta e^{-\frac{x}{2}}-\left(t+c_{2}\right) e^{-x}$ \\
6 & $6(\varepsilon=-1)$ & $u=c_{1}+c_{2} e^{-x}+\delta x-t$ \\
$7^{\mathrm{c}}$ & $7\left(\varphi^{\prime}=\right.$ const $)$ & $u=c_{1}+\varepsilon t+\frac{1}{2 k}\left(\delta \sqrt{1-4 \varepsilon k^{2}}-1\right)\left(x+\frac{1}{k} t\right)$ \\
$8^{\mathrm{d}}$ & $8(\varepsilon=-1)$ & $u=c_{1}+c_{2} e^{-x}-\left(1+\frac{1}{k} e^{-x}\right) t+$ \\
& & $+\frac{\delta}{k}\left[\left(k-\frac{1}{2} e^{-x}\right) x-3 \sqrt{k\left(k+e^{-x}\right)}+\left(2 k-e^{-x}\right) \log \left(\sqrt{k}+\sqrt{k+e^{-x}}\right)\right]$ \\
$9^{\mathrm{e}}$ & $8(\varepsilon=-1)$ & $u=c_{1}+c_{2} e^{-x}-\left(1-\frac{1}{k} e^{-x}\right) t+$ \\
& & $+\frac{\delta}{k}\left[\left(k+\frac{1}{2} e^{-x}\right) x-3 \sqrt{k\left(k-e^{-x}\right)}+\left(2 k+e^{-x}\right) \log \left(\sqrt{k}+\sqrt{k-e^{-x}}\right)\right]$ \\
$10^{\mathrm{f}}$ & $8(\varepsilon=1)$ & $u=c_{1}+c_{2} e^{-x}+\left(1-\frac{1}{k} e^{-x}\right) t+$ \\
& & $+\frac{\delta}{k}\left[2\left(k+\frac{1}{2} e^{-x}\right) \arctan \sqrt{\frac{1}{k}\left(e^{-x}-k\right)}-3 \sqrt{k\left(e^{-x}-k\right)}\right]$ \\
$11^{\mathrm{g}}$ & $7\left(\varphi^{\prime} \neq \mathrm{const}\right)$ & $w^{\prime}(z)=1-\varepsilon k^{2} \frac{z}{w(z)}$ \\
12 & 9 & $w^{\prime}(z)=\frac{1}{w(z)}-\sqrt[3]{\frac{4}{3 z}}$ \\
$13^{\mathrm{h}}$ & 10 & $w^{\prime}(\varphi)=\frac{\sqrt{\varphi-k w(\varphi)}}{w(\varphi)}-1$ \\
14 & 11 & $w^{\prime}(z)=1-\varepsilon \frac{e^{-2 z}}{w(z)}$ \\
\hline
\end{tabular}

${ }^{\mathrm{a}}$ In this column, the numbers of algebras from Table 1 are indicated.

${ }^{\mathrm{b}}$ In this column, $\varepsilon, \delta \in\{1,-1\} ; c_{1}, c_{2}$ are arbitrary real constants.

${ }^{\mathrm{c}}$ In this case, $k \neq 0$, if $\varepsilon=-1$, and $0<|k| \leq \frac{1}{2}$, if $\varepsilon=1$.

${ }^{\mathrm{d}}$ In this case, $0<k<1$.

${ }^{\mathrm{e}}$ In this case, $0<k<1, x \geq-\log k$.

${ }^{\mathrm{f}}$ In this case, $0<k<1, x \leq-\log k$.

${ }^{\mathrm{g}}$ In this case, $k \neq 0$.

${ }^{\mathrm{h}}$ In this case, $k \neq 0,-1$. 
Table 3: The exact solutions of equation 3 with $c=0$

\begin{tabular}{|c|c|c|}
\hline No. & Sol. ${ }^{\mathrm{a}}$ & Exact solution ${ }^{\mathrm{b}}$ \\
\hline 1 & 2 & $u=c_{1}+\frac{a}{2 b} x\left(c_{2}+\frac{a}{2} t-\log x\right)$ \\
\hline 2 & 5 & $u=c_{1}-t+4 \delta \sqrt{\frac{x}{b}}+\frac{a}{2 b} x\left(c_{2}+\frac{a}{2} t-\log x\right)$ \\
\hline 3 & 6 & $u=c_{1}+\frac{a+2 \varepsilon}{2 b} x\left(c_{2}+\frac{a-2 \varepsilon}{2} t-\log x\right)$ \\
\hline 4 & 3 & $u=\varepsilon c_{1}^{2} e^{-\varepsilon t}+4 \delta c_{1} e^{-\frac{\varepsilon}{2} t} \sqrt{\frac{x}{b}}+\frac{a+2 \varepsilon}{2 b} x\left(c_{2}+\frac{a-2 \varepsilon}{2} t-\log x\right)$ \\
\hline $5^{\mathrm{c}}$ & 7 & $u=\frac{x}{b}\left[c_{1}+\left(\varepsilon+\frac{a^{2}}{4}\right) t-\frac{a}{2} \log x+\frac{1}{2 k}\left(\delta \sqrt{1-4 \varepsilon k^{2}}-1\right)\left(\frac{1}{k} t+\log x\right)\right.$ \\
\hline $6^{\mathrm{d}}$ & 8 & $\begin{array}{l}u=c_{1}-\frac{1}{k} t+\frac{x}{b}\left\{c_{2}+\left(\frac{a^{2}}{4}-1\right) t-\frac{a}{2} \log x+\right. \\
\left.+\delta\left[\left(1-\frac{b}{2 k x}\right) \log \left(\frac{2 k x}{b}\left(1+\sqrt{1+\frac{b}{k x}}\right)+1\right)-3 \sqrt{1+\frac{b}{k x}}\right]\right)\end{array}$ \\
\hline $7^{\mathrm{e}}$ & 9 & $\begin{array}{l}u=c_{1}+\frac{1}{k} t+\frac{x}{b}\left\{c_{2}+\left(\frac{a^{2}}{4}-1\right) t-\frac{a}{2} \log x+\right. \\
+\delta\left[\left(1+\frac{b}{2 k x}\right) \log \left(\frac{2 k x}{b}\left(1+\sqrt{1-\frac{b}{k x}}\right)-1\right)-3 \sqrt{1-\frac{b}{k x}}\right]\end{array}$ \\
\hline $8^{\mathrm{f}}$ & 10 & $\begin{array}{l}u=c_{1}-\frac{1}{k} t+\frac{x}{b}\left\{c_{2}+\left(\frac{a^{2}}{4}+1\right) t-\frac{a}{2} \log x+\right. \\
+\delta\left[2\left(1+\frac{b}{2 k x}\right) \arctan \sqrt{\frac{b}{k x}-1}-3 \sqrt{\frac{b}{k x}-1}\right]\end{array}$ \\
\hline
\end{tabular}

\footnotetext{
${ }^{\mathrm{a}}$ In this column, the numbers of solutions of equation 60 from Table 2 are indicated.

${ }^{\mathrm{b}}$ In this column, $\varepsilon, \delta \in\{1,-1\} ; c_{1}, c_{2}$ are arbitrary real constants.

${ }^{\mathrm{c}}$ In this case, $k \neq 0$, if $\varepsilon=-1$, and $0<|k| \leq \frac{1}{2}$, if $\varepsilon=1$.

${ }^{\mathrm{d}}$ In this case, $0<k<1$.

${ }^{\mathrm{e}}$ In this case, $0<k<1, x \geq \frac{b}{k}$.

${ }^{\mathrm{f}}$ In this case, $0<k<1, x \leq \frac{b}{k}$.
}

7) ODE 13 is obtained, if we put in ODE 10 from Table $11 w(\varphi)=\varphi^{\prime}$;

8) ODE 14 is obtained, if we put in ODE 11 from Table 1

$$
z=\frac{1}{2} y, \quad \omega=e^{-\frac{1}{2} y} \sqrt{\varphi^{\prime}(y)}
$$

\section{Exact solutions of equation (3)}

Using solutions 2-3, 5-10 of equation (6) (see Table 2) and the point transformations of variables (4)-(5), we obtain a number of exact solutions of equation (3) presented in Tables 3 and 4

Compare solutions obtained by us with the solutions found in 9 . Changing 
Table 4: The exact solutions of equation $(3)$ with $c \neq 0$

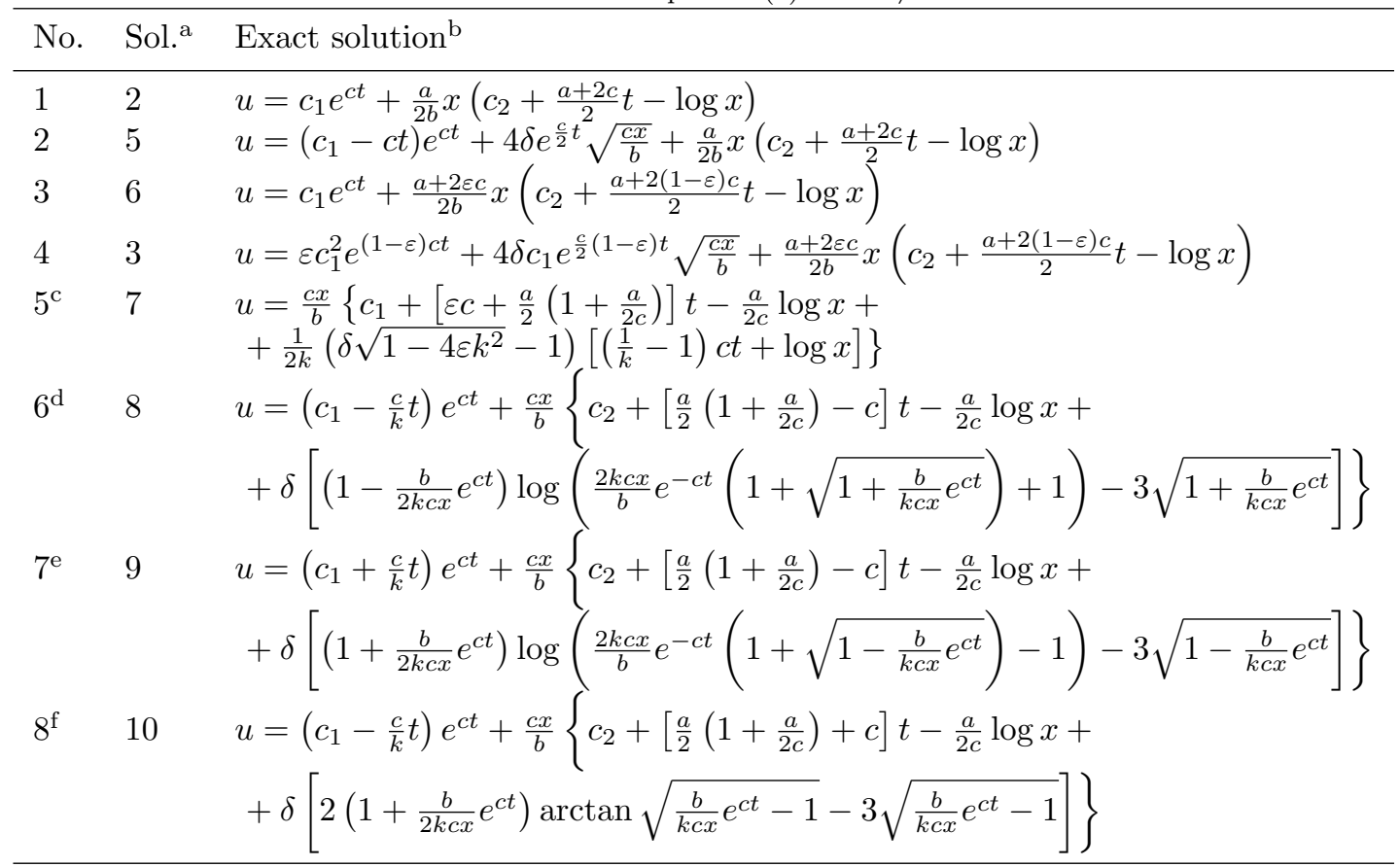

${ }^{\mathrm{a}}$ In this column, the numbers of solutions of equation $\sqrt{6}$ from Table 2 are indicated.

${ }^{\mathrm{b}}$ In this column, $\varepsilon, \delta \in\{1,-1\} ; c_{1}, c_{2}$ are arbitrary real constants.

${ }^{\mathrm{c}}$ In this case, $k \neq 0$, if $\varepsilon=-1$, and $0<|k| \leq \frac{1}{2}$, if $\varepsilon=1$.

${ }^{\mathrm{d}}$ In this case, $0<k<1$.

${ }^{\mathrm{e}}$ In this case, $0<k<1, x \geq \frac{b}{k c} e^{c t}$.

${ }^{\mathrm{f}}$ In this case, $0<k<1, x \leq \frac{b}{k c} e^{c t}$. 
constants, we can rewrite the Bobrov solutions in the following form:

$$
\begin{gathered}
u(t, x)=c_{1}+c_{3} x\left\{c_{2}+\left(a-b c_{3}\right) t-\log x\right\} \\
u(t, x)=c_{1}+c_{3} t+x\left\{c_{2}+c_{4} t-\frac{a}{2 b} \log x-\frac{3 \delta \sqrt{-c_{3} b K}}{b} \sqrt{1+\frac{1}{K x}}-\right. \\
\left.-\frac{\delta c_{3} K}{\sqrt{-c_{3} b K}}\left(1-\frac{1}{2 K x}\right) \log \left[2 K x\left(1+\sqrt{1+\frac{1}{K x}}\right)+1\right]\right\}, \\
K=\frac{4 c_{4} b-a^{2}}{4 c_{3} b}, \delta= \pm 1 .
\end{gathered}
$$

It is easy to see that solutions 1 and 3 (and also 5 with $a=1$ ) from Table 3 are of the form (9), and solution 6 is of the form 10 .

\section{Applications to solving various BVPs with the governing PDE (3)}

In this section we are going to apply the solutions of the non-linear BlackSholes equation (3) found in Section 4 to solving various BVPs.

In [7] the following stationary BVP for the equation

$$
\frac{1}{2} \tilde{\sigma}^{2} S^{2} \frac{\partial^{2} V}{\partial S^{2}}+b \sigma^{2} S^{3}\left(\frac{\partial^{2} V}{\partial S^{2}}\right)+r\left(\frac{\partial V}{\partial S} S-V\right)=0, \quad S \in(c, d)
$$

under the Dirichlet boundary conditions

$$
V(c)=V_{c}, \quad V(d)=V_{d}
$$

for some fixed $d>c>0$ was considered. In equation (11) the parameter $\tilde{\sigma}^{2}$ is as follows

$$
\tilde{\sigma}^{2}=\sigma^{2}\left(1-\frac{a}{\sigma} \sqrt{\frac{2}{\pi d t}}\right) .
$$

The authors proved that under some conditions on the constants $c, d, V_{c}$, and $V_{d}$ the BVP (11) and (12) admits a convex unique classic solution, which can be obtained as the limit of a non-increasing (respectively non-decreasing) sequence of upper (lower) solutions.

Note that in the case $a=0,(11)$ coincides with the stationary version of equation (2). So, it is convenient here to consider the following stationary BVP

$$
\begin{aligned}
& a x^{2} u_{x x}+b x^{3} u_{x x}^{2}+c x u_{x}-c u=0, \quad x \in(A, B), \\
& \quad x=A: u=U_{A} \\
& \quad x=B: u=U_{B}
\end{aligned}
$$

where $a>0, b>0, c>0, B>A \geq 0, U_{A} \geq 0$, and $U_{B}>0$. We are going to find an exact solution of the BVP $[13-(15)$ in explicit form. 
Consider solution 5 of Table 4

$$
\begin{aligned}
u=\frac{c x}{b}\left\{c_{1}+[\varepsilon c+\right. & \left.\frac{a}{2}\left(1+\frac{a}{2 c}\right)\right] t-\frac{a}{2 c} \log x+ \\
& \left.+\frac{1}{2 k}\left(\delta \sqrt{1-4 \varepsilon k^{2}}-1\right)\left[\left(\frac{1}{k}-1\right) c t+\log x\right]\right\},
\end{aligned}
$$

where $c_{1} \in \mathbb{R}, \varepsilon, \delta \in\{-1,1\}$, and $k \neq 0$ if $\varepsilon=-1$ or $0<|k| \leq \frac{1}{2}$ if $\varepsilon=1$.

This is a solution of the evolution equation (3). To obtain a solution of the stationary one (13), we need to eliminate the time coefficient in formula (16), i.e.

$$
\varepsilon c+\frac{a}{2}\left(1+\frac{a}{2 c}\right)+\frac{c}{2 k}\left(\delta \sqrt{1-4 \varepsilon k^{2}}-1\right)\left(\frac{1}{k}-1\right)=0 .
$$

Solving this equation w.r.t. the parameter $k$, we get ${ }^{3}$

$$
k_{1,2}=\frac{c}{2} \cdot \frac{\varepsilon c-\frac{a}{2}\left(1+\frac{a}{2 c}\right) \pm\left|\varepsilon c+\frac{a}{2}\left(1+\frac{a}{2 c}\right)\right| \sqrt{1+\frac{2 a}{c}\left(1+\frac{a}{2 c}\right)}}{(1+\varepsilon) c^{2}+\varepsilon a c\left(1+\frac{a}{2 c}\right)+\frac{a^{2}}{4}\left(1+\frac{a}{2 c}\right)^{2}} .
$$

In this cases, the relevant equation $(13)$ admits such solution:

$$
u=\frac{c x}{b}\left(c_{1}+M \log x\right),
$$

where $M \equiv \frac{1}{2 k}\left(\delta \sqrt{1-4 \varepsilon k^{2}}-1\right)-\frac{a}{2 c}=\frac{\varepsilon+\frac{a}{2 c}\left(1+\frac{a}{2 c}\right)}{1-\frac{1}{k}}-\frac{a}{2 c}$, i.e.,

$$
M_{1,2}=\frac{\varepsilon+\frac{a}{2 c}\left(1+\frac{a}{2 c}\right)}{1-\frac{2}{c} \cdot \frac{(1+\varepsilon) c^{2}+\varepsilon a c\left(1+\frac{a}{2 c}\right)+\frac{a^{2}}{4}\left(1+\frac{a}{2 c}\right)^{2}}{\varepsilon c-\frac{a}{2}\left(1+\frac{a}{2 c}\right) \pm\left|\varepsilon c+\frac{a}{2}\left(1+\frac{a}{2 c}\right)\right| \sqrt{1+\frac{2 a}{c}\left(1+\frac{a}{2 c}\right)}}}-\frac{a}{2 c} .
$$

Substitute solution (17) into the boundary conditions (14) and (15), putting in (14) $A=0$ and $U_{A}=0$. Then (14) is satisfied in the sense of the right limit in $x=0$, and from 15 we obtain the following condition on the coefficient $c_{1}$ :

$$
\frac{c B}{b}\left(c_{1}+M \log B\right)=U_{B} .
$$

Thus, we get that

$$
c_{1}=\frac{b U_{B}}{c B}-M \log B .
$$

Hence, we proved the following statement.

Proposition 2. The $B V P(13)-15$ with $A=0$ and $U_{A}=0$ admits the classical solution (17) on $x \in(0, B)$, where the constants $M$ and $c_{1}$ are defined by formulae (18) and 19), respectively.

\footnotetext{
${ }^{3}$ It should be noted that here $k \neq 0$, if $\varepsilon=-1$, and $0<|k| \leq \frac{1}{2}$, if $\varepsilon=1$ (see Table 4.
} 
The slightly different result is obtained in the case $A>0$. Here the boundary conditions (14) and (15) give

$$
\left\{\begin{array}{l}
\frac{c A}{b}\left(c_{1}+M \log A\right)=U_{A}, \\
\frac{c B}{b}\left(c_{1}+M \log B\right)=U_{B} .
\end{array}\right.
$$

From the second condition we still receive formula $(19)$, but the first one leads to the condition

$$
M \log \frac{A}{B}+\frac{b}{c}\left(\frac{U_{B}}{B}-\frac{U_{A}}{A}\right)=0 .
$$

Thus, the following statement is obtained.

Proposition 3. The BVP (13)-15 with $A>0$ admits the classical solution (17) on $x \in(A, B)$, where the constants $M$ and $c_{1}$ are defined by formulae (18) and (19), respectively, if and only if condition (20) holds.

Now we are going to consider an evolution BVP with the governing equation (3) on $x \in(0,+\infty)$ and $t \in(0, T)$, the terminal condition

$$
t=T: u=h(x),
$$

and the boundary condition

$$
x=0: u=0 .
$$

In the terminal condition, $h(x)$ is the so-called pay-off function, which traditionally is taken in the form

$$
h(x)=(x-K)^{+},
$$

and

$$
h(x)=(K-x)^{+},
$$

for the European Call and Put options, respectively. Here $K>0$ is some real constant and the designation

$$
f^{+}(x)= \begin{cases}f(x) & \text { if } f(x)>0 \\ 0 & \text { if } f(x) \leq 0\end{cases}
$$

is used.

Note that formulae 21) and (22) are the simplest forms of the pay-off, which have the strong economic sense, but there are no any evidences against using others, more sophisticated, pay-off functions.

In our investigation we deals with the following pay-off $h(x)$ :

$$
h(x)=[A x(B+\log x)]^{+},
$$




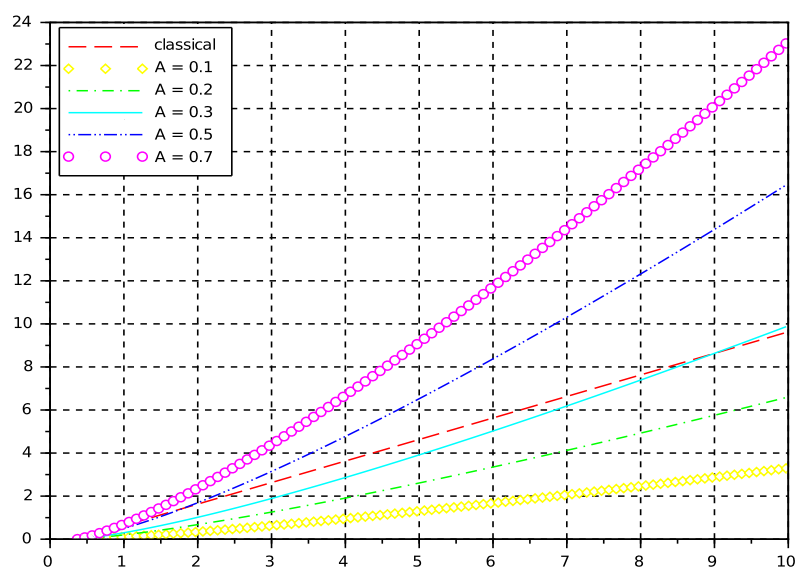

Figure 1: Graphs of pay-off functions 21 for $K=\frac{1}{e}$, and 23 for $B=1$ and various values of $A$

where $A>0$ and $B$ are some real constants. It is easy to see that the behavior of function (23) is very close to behavior of the classical pay-off function for the European Call option (21) (see Fig. 11).

Thus, we are dealing with such European Call option type BVP

$$
\begin{aligned}
& u_{t}+a x^{2} u_{x x}+b x^{3} u_{x x}^{2}+c x u_{x}-c u=0, x \in(0,+\infty), t \in(0, T), \\
& \quad t=T: u=[A x(B+\log x)]^{+}, \\
& x=0: u=0
\end{aligned}
$$

where $a>0, b>0, c>0, A>0$, and $B$ are some real constants.

We again intend to use solution 5 of Table 4 (see formula $(16)$ ). For convenience, we rewrite this solution in the form

$$
u=\frac{c x}{b}\left(C_{1}+M(t-T)+N \log x\right),
$$

where

$$
\begin{gathered}
M=\varepsilon c+\frac{a}{2}\left(1+\frac{a}{2 c}\right)+\frac{c}{2 k}\left(\delta \sqrt{1-4 \varepsilon k^{2}}-1\right)\left(\frac{1}{k}-1\right), \\
N=\frac{1}{2 k}\left(\delta \sqrt{1-4 \varepsilon k^{2}}-1\right)-\frac{a}{2 c}
\end{gathered}
$$

and

$$
C_{1}=c_{1}+M T \text {. }
$$

We also should remind the readers that in $27 k \neq 0$, if $\varepsilon=-1$, and $0<|k| \leq \frac{1}{2}$, if $\varepsilon=1$. 
In view of the terminal condition 225, we are looking for a solution of the BVP 24)-26) in the form

$$
u= \begin{cases}\frac{c x}{b}\left(C_{1}+M(t-T)+N \log x\right) & \text { if } x>e^{-B+\frac{M}{N}(T-t)}, \\ 0 & \text { if } 0<x \leq e^{-B+\frac{M}{N}(T-t)} .\end{cases}
$$

Substituting formula 28 in the terminal condition 25 and the boundary one (26), we find that

$$
k=-\frac{2 c(2 b A+a)}{(2 b A+a)^{2}+4 \varepsilon c^{2}},
$$

and

$$
C_{1}=B N
$$

Thus, we proved the statement.

Proposition 4. The European Call option type BVP (24)-(26) admits the classical solution

$$
u= \begin{cases}\frac{c x}{b}(B N+M(t-T)+N \log x) & \text { if } x>e^{-B+\frac{M}{N}(T-t)}, \\ 0 & \text { if } 0<x \leq e^{-B+\frac{M}{N}(T-t)},\end{cases}
$$

where

$$
\begin{gathered}
M=\varepsilon c+\frac{a}{2}\left(1+\frac{a}{2 c}\right)+\frac{c}{2 k}\left(\delta \sqrt{1-4 \varepsilon k^{2}}-1\right)\left(\frac{1}{k}-1\right), \\
N=\frac{1}{2 k}\left(\delta \sqrt{1-4 \varepsilon k^{2}}-1\right)-\frac{a}{2 c},
\end{gathered}
$$

and $k$ is defined by formula 29 obeying the additional condition: $k \neq 0$, if $\varepsilon=-1$, and $0<|k| \leq \frac{1}{2}$, if $\varepsilon=1$.

Note that

$$
u \sim x \log x \quad \text { as } x \rightarrow+\infty .
$$

Example. Let $a=2 \cdot 10^{-2}, b=4 \cdot 10^{-6}, c=10^{-1}, \delta=1, \varepsilon=-1$. If we put $A=10^{4}, B=1$, and $T=1$ (one year) then $k=\frac{2}{3}, M=-0.064, N=0.4$, and

$$
u(t, x)= \begin{cases}400 x(25 \log x-4 t+29) & \text { if } x>e^{0.16 t-1.16} \\ 0 & \text { if } 0<x \leq e^{0.16 t-1.16}\end{cases}
$$

Graph of this solution is presented on Fig. 2.

\footnotetext{
${ }^{4}$ Note that our parameters $a, b, c$, and $T$ are similar to the ones in 15 p. 809].
} 


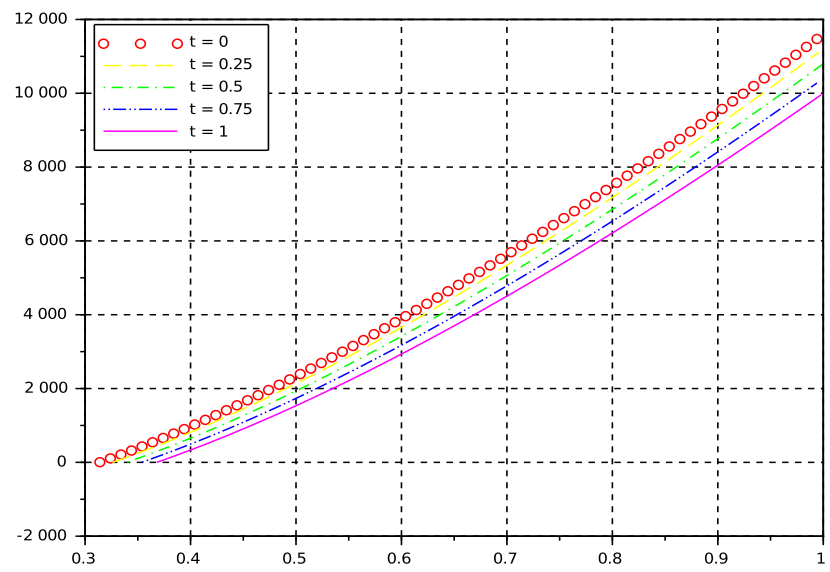

Figure 2: Solution of the BVP $(24)-(26) u=u\left(t_{i}, x\right)$, for some fixed values $t_{i}$

\section{Conclusions}

In this article, we investigated the non-linear Black-Scholes equation (2) from the group theoretic point of view.

First, in Section 2 using the point transformations of variables (4)-(5), we reduced the equation to the more simple and canonical form (6). We found that for this equation there are known several exact solutions. Our main purpose was to carry out the symmetry analysis of the equation in order to obtain a comprehensive list of self-similar (invariant) exact solutions of the one using the method of symmetry reduction to the ordinary differential equations.

In Section 3, we found the MAI of equation (6). This algebra is the fivedimensional Lie one, which can be written as a semi-direct sum of a onedimensional algebra and a four-dimensional solvable ideal. Taking into account the widely known classification of sub algebras of low dimensional Lie algebras 13 and using the Patera-Winternitz-Zassenhaus algorithm, we found the optimal system of one-dimensional sub-algebras of MAI of equation (6). Using the ones, which satisfy the necessary conditions of existence of the non-degenerate invariant solutions, we carried out the symmetry reduction of the equation to the ordinary differential equations of the first and second order (see Table 1) and found several general solutions of the ones. For a number of the reduced equations we could not find the general solutions in the explicit form in elementary functions (see Cases 11-14 in Table 2).

Using the obtained general solutions of the reduced equations, in Section 4 , we constructed a set of exact solutions of the Black-Scholes equation under study. The complete list of the solutions is presented in Tables 3 and 4 . Finally, 
we compared our solutions with the ones found previously.

In Section 5, we applied results found in the previous section for solving several BVPs with the governing Black-Scholes equation (3) in the case $c>0$. We utilized solution 5 of Table 4 to find exact classical solutions of both the stationary (13)-15 and non-stationary (24)-26) BVPs of the Dirichlet and European Call option types, respectively.

\section{Funding}

This research did not receive any specific grant from funding agencies in the public, commercial, or not-for-profit sectors.

\section{References}

\section{References}

[1] Black F, Scholes M. The pricing of options and corporate liabilities. J Political Econ. 1973;81:637-59.

[2] Merton RC. Theory of rational option pricing. Bell J Econ. 1973;4:141-83.

[3] Agliardi R, Popivanov P, Slavova A. On nonlinear Black-Scholes equations. Nonlinear Anal Differ Equ 2013;1(2):75-81.

[4] Bordag LA, Frey R. Nonlinear option pricing models for illiquid markets: scaling properties and explicit solutions. In: Ehrhardt M, editor. Nonlinear Models in Mathematical Finance: New Research Trends in Option Pricing, New York: Nova Science Publishers Inc.; 2008.

[5] Çetin U, Jarrow RA, Protter P. Liquidity risk and arbitrage pricing theory. Finance Stoch 2004;8:311-41.

[6] Frey R, Polte U. Nonlinear Black-Scholes equation in finance: associated control problems and properties of solutions. SIAM J Control Optim 2011;49(1):185-204.

[7] Amster P, Averbuj CG, Mariani MC, Rial D. A Black-Scholes option pricing model with transaction costs. J Math Anal Appl 2005;303:688-95.

[8] Bakstein D., Howison S. A non-arbitrage liquidity model with observable parameters for derivatives. Oxford University Preprint 2003.

[9] Bobrov MV. The fair price valuation in illiquid markets. Master's Thesis in Financial Mathematics, Technical report IDE0738, Halmstad University, Sweden, 2007.

[10] Polyanin AD, Zaitsev VF. Handbook of nonlinear partial differential equations. Boca Raton: CRC Press; 2012. 
[11] Head AK. LIE, a PC program for Lie analysis of differential equations. Comput Phys Commun 1996;96:311-13.

[12] Basarab-Horwath P, Lahno V, Zhdanov R. The structure of Lie algebras and the classification problem for partial differential equations. Acta Appl Math 2001;69:43-94.

[13] Patera J, Winternitz P. Subalgebras of real three- and four-dimensional Lie algebras. J Math Phys 1977;18:1449-55.

[14] Polyanin AD, Zaitsev VF. Handbook of exact solutions for ordinary differential equations. Boca Raton: CRC Press; 2003.

[15] Ankudinova J, Ehrhardt M. On the numerical solutions of nonlinear BlackScholes equations. Comput Math Appl 2008;56:799-812. 